\title{
THE EMISSION OF X-RAYS BY FISSION FRAGMENTS
}

\author{
H.C. GRIFFIN \\ Department of Chemistry, University of Michigan, \\ Ann Arbor, MI 48109-1055 (USA)
}

(Received January 10, 1990)

\begin{abstract}
Approximately $5 \%$ of the $\boldsymbol{y}$-ray transitions in fission fragments are internally converted, giving rise to $X$-rays. $X$-ray energies are characteristic of $Z$, but yields depend strongly and not smoothly on $\mathrm{Z}$ and $\mathrm{A}$ of the fragments and on time. If these biases can be accepted, $\mathrm{X}$-rays can be used to identify the atomic number of fission fragments. More generally, internal conversion and $\mathrm{X}$-ray emission must be interpreted in the context of detailed knowledge of $\gamma$-ray transitions.
\end{abstract}

\section{Introduction}

Soon after VorTOVETSKII, LEVIN and MARCHENKo, 1 and SKLIAREVSKII, FOMENKo and STEPANOV 2 detected $\mathrm{K} X$ rays among the prompt emissions from fission fragments, CARTER, WAGNER and WYMAN ${ }^{3}$ measured the spectra of $L$ and $K$ $\mathrm{X}$-rays associated with neutron induced fission of ${ }^{235} \mathrm{U}$ in an attempt to determine the division of nuclear charge between fission fragments. Interpretation of their low resolution spectra (from a gas-flow proportional counter for $\mathrm{LX}$-rays and a $\mathrm{Na}(\mathrm{Ti}$ ) scintillator for $\mathrm{K} \mathrm{X}$-rays) was based on the expectation that some electron vacancies would result from the fission process itself and yields from this mechanism would not depend strongly on $\mathrm{Z}$ and $\mathrm{A}$ of the fragments. It was this expectation that fission would produce X-rays in the fragments that led to particular interest in X-ray emission in fission. However, GLENDENIN and GRIFFIN ${ }^{4}$ showed that internal conversion accounts for essentially all of the $\mathrm{K} X$-rays and that the yields have considerable dependence on $\mathrm{Z}$ and $\mathrm{A}$.

In spite of disapointment that X-rays are not an unbiased measure of atomic number of fission fragments, many instrumental studies of fission carried out during the 1960's included X-ray detectors for $Z$ identification. Most often semiconductor detectors were used; the individual elements could be resolved without depending on assumptions required for deconvolution of poor resolution spectra. These improved detectors were also used to explore details in the yields of X-rays for several fissioning systems. High resolution $\gamma$-ray detectors, used in conjunction with other 
parameters, permitted detection of individual transitions in fragments and led to an emphasis on spectroscopy.

The vigorous activity of 1966-1971 was followed by fifteen years of relative inactivity in measurements of fission X-rays. PHILLIPS et al. ${ }^{5}$ resumed the emphasis on spectroscopy by applying techniques of inbeam spectroscopy to the $\gamma$-ray transitions (and associated features such as internal conversion and X-ray emission) in specific fragments from ${ }^{252} \mathrm{Cf}$ fission. These new studies derive from the utility of multi-detector arrays and sophisticated analysis techniques, which permit the study of fission in the manner of a decay scheme.

Several of the major developments in characterizing and using X-rays emitted by fission fragments are given in Table 1. Addition details are given in the following sections.

\section{Identification of fission X-rays}

Conjectures on the emission of $\mathrm{X}$-rays by fission fragments probably originated in questions concerning what happens to the two $\mathrm{K}$ electrons and eight $\mathrm{L}$ electrons of $\mathrm{a}$ heavy atom as it fissions and the system is transformed into two atoms which collectively have twice as many electrons in these shells. This question is too simplistic; BOHR ${ }^{17}$ calculated that few $K$ vacancies are expected either from the fission act or during stopping of fragments. However a process similar to electron "shakeoff" in radioactive decay 18 could be significant. In any event characterization of the electromagnetic radiations emitted during fission awaited radiation spectrometers of good efficiency (required for coincidence experiments in a complex radiation environment) and adequate resolution. The detector which opened the field of investigation was the $\mathrm{NaI}(\mathrm{TI})$ scintillator. The first detailed measurements ${ }^{19}$ of prompt electromagnetic radiations was made with ${ }^{252} \mathrm{Cf}$; the clean environment of spontaneous fission as opposed to thermal neutron induced fission was chosen to simplify the measurements. These measurements emphasized intermediate energies and showed little structure. A small peak at about $60 \mathrm{keV}$ was reported, but SKLIAREVSKII et al. ${ }^{2}$ identified such a peak as due to inelastic scattering of neutrons from iodine in the scintillation detector. 
Table 1.

Major developments in characterizing and using X-rays emitted by fission fragments

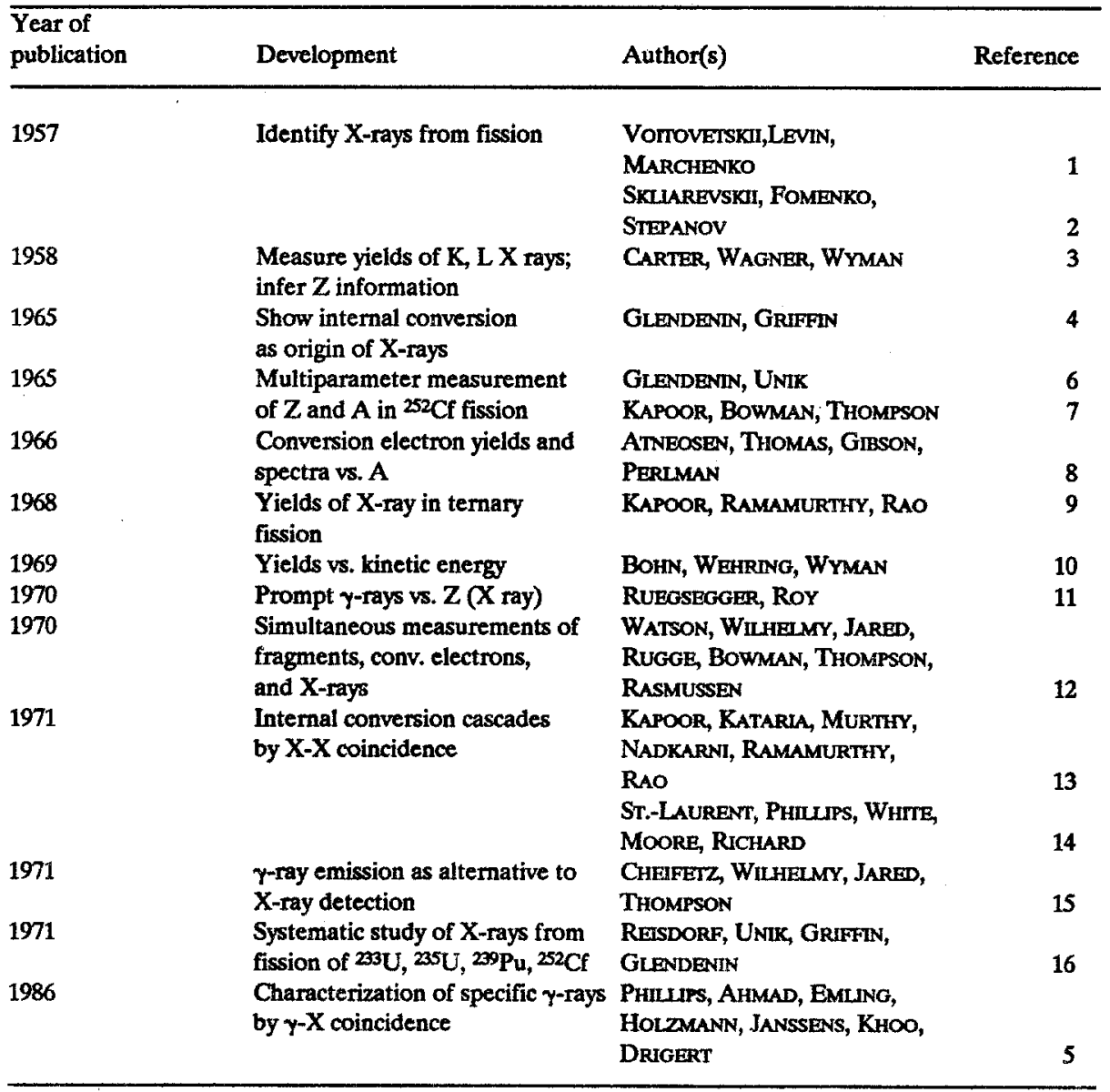

Measurements ${ }^{1,2}$ which clearly showed $\mathrm{K}$ X-rays from ${ }^{236} \mathrm{U}$ fission fragments were reported soon. The measurements of SkJIAREVSKII et al. ${ }^{2}$ are particularly important. They used $\mathrm{a} \mathrm{Pb}$ shield to distinguish neutrons from photons and found that a considerable fraction of the X-rays are emitted more than a nanosecond after fisssion. They cited similar results for ${ }^{234} \mathrm{U}$ and ${ }^{240} \mathrm{Pu}$, and concluded that internal conversion is responsible for much of the $\mathrm{X}$-ray emission. (Their measurements did not rule out contributions from other mechanisms.) In any event, their work was followed by measurements, also with thermal neutron induced fission of ${ }^{235} \mathrm{U}$, of the 
spectra of $\mathbf{L} X$-rays (with a gas proportional counter) as well as $\mathrm{K} \mathrm{X}$-rays. ${ }^{3}$ Although the resolution of these spectra was not adequate to show individual elements, the spectra were interpreted in terms of an average atomic number for the light and heavy groups. This interpratation disagreed with radiochemical measurements from which the most probable nuclear charge as a function of product mass number $\left(Z_{p}\right)$ had been inferred. The major difficulty with radiochemical measurements is the very short half lives for $\beta$-decay of the primary fission products (fission fragments after $\gamma$ deexcitation). These $\mathrm{X}$-ray spectra were the first measurements of nuclear charge which avoided this difficulty, and it was not clear which result was correct.

Measurements ${ }^{4}$ of the time of emission for intervals as short as 0.1 ns showed that essentially all of the X-rays are delayed compared to normal lifetimes of inner vacancies. That the vacancies arise from internal conversion, and therefore are delayed by times corresponding to lifetimes for $\gamma$-ray emission, was supported by detection of electrons in coincidence with fission $(1.0 \pm 0.2$ electrons per fission) and by showing that $\mathrm{X}$-ray intensities followed the general patern of internal conversion.

Therefore interpretation of the intensity of fission $X$-rays depends on knowledge of fission $\gamma$-rays. The early $\gamma$-ray measurements used low resolution detectors - at least in comparison with the average interval between $\gamma$-rays - and treated the spectra as continuous. It was expected that the large number and great variety of $\gamma$-rays emitted in the deexcitation of fission fragments would justify the assumption that internal conversion, averaged over a range of isotopes comparable to the mass resolution of multiparameter measurements, would be a smooth function of $A$ for each Z. Many measurements were made and analyzed with these assumptions. As $\gamma$-ray spectroscopy improved, it become clear that this assumption was not justified.

Although not a typical X-ray emitting nuclide and not characterized until after most of the fission X-ray studies, ${ }^{134} \mathrm{Te}$ illustrates the extreme variation to be expected in X-ray yield. The probability that a tellurium fragment produced in low energy fission will emit a prompt ( $<1 \mathrm{~ns}$ ) X-ray is about $2 \% .{ }^{16}$ About half of the deexcitations of the $0.16-\mu \mathrm{s} 6+$ state in ${ }^{134} \mathrm{Te}$ produce an $\mathrm{X}$-ray, and ${ }^{134} \mathrm{Te}$ is produced in high yield. For times longer than a few nanoseconds, any selections of tellurium fragments based on X-ray emission will be dominated by ${ }^{134} \mathrm{Te}$ regardless of substantial shifts in yield among the isotopes. 


\section{Characterization of fission X-ray}

Most of the early studies emitted in fission (e.g., GLENDENIN and UNIK, 6 and KAPOOR, BOWMAN and THOMPSON7) involved both characterizing the emission process and using $X$-rays as a probe, such as to determine nuclear charge or infer multipolarities of $\gamma$ transitions. In retrospect it sufficient to know the mechanism for producing electron vacancies and the insensitivity of the energy or decay mode of these vacancies to ionization of the outer shells. However, the $\boldsymbol{\gamma}$-ray transitions were not well understood and it appeared simpler to investigate the $\mathrm{X}$-rays directly.

A typical arrangement of target (or fission source) and detectors is given in Fig. 1. With this " $90^{\circ}$ " geometry Doppler shifts disperse X-ray preserved good electron energy resolution by using magnetic steering. They identified ${ }^{108} \mathrm{Tc}$ (69 keV conversion electrons), ${ }^{136} \mathrm{I}(59 \mathrm{keV}),{ }^{140} \mathrm{Cs}(78 \mathrm{keV})$, and ${ }^{146} \mathrm{La}(64 \mathrm{keV})$ as major Xray and $\mathrm{e}^{-}$emitters, thus confirming the importance of doubly odd nuclides for $\mathrm{X}$-ray emission that was earlier inferred ${ }^{6}$ from less direct data.

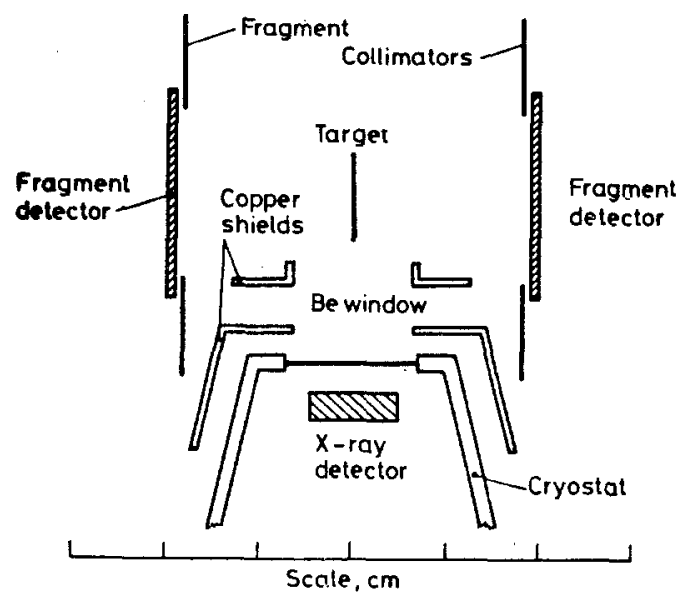

Fig. 1. Typical arrangement for detecting $\mathrm{X}$-rays and dynamical properties of both fragments in fission (taken from Ref. 20.)

Although generally not involving detection of $\mathrm{X}$-rays, several studies of $\gamma$ transitions in fission fragments substantially enhanced our understanding of X-ray emission. JOHN, GUY and WESOLOWSKI, 22 and CIARK, GLENDENIN and TALBERT ${ }^{23}$ studied isomeric transitions in ${ }^{252} \mathrm{Cf}$ fission fragments. Since isomeric transitions generally have substantial internal conversion probabilities, these studies emphasized 
those nuclides which might have dominated $\mathrm{X}$-ray measurements when acquisition times were not constrained to a few nanoseconds.

BOHN, WEHRING and WYMAN ${ }^{10}$ performed 3-parameter measurements ( $X$-rays in a Nal detector; fragments in Si detectors) of the relation between fragment kinetic energy and X-ray emission probability, but their technique did not correct for variations of average kinetic with mass. WATSON, JARED and THOMPSON24 performed similar measurements (among others), but used a $\mathrm{Si}(\mathrm{Li})$ detector capable of resolving individual elements and were able to conclude that even- $Z$ fragments are enhanced at high kinetic energies. Using the apparatus shown in Figs. 1 and 2, REISDORF, UNIK, GRIFFIN and GLENDENIN ${ }^{16,20}$ sought to determine the isotopic dependence of X-ray emission probability and measure charge distribution and dispersion for four fissioning systems (thermal neutron induced fission of ${ }^{233} \mathrm{U}$, ${ }^{235} \mathrm{U}$ and ${ }^{239} \mathrm{Pu}$, and spontaneous fission of ${ }^{252} \mathrm{Cf}$ ). Their analysis assumed that $\mathrm{X}$-ray emission probability is a linear function of mass number for each energies but cause little shift, and detection efficiencies are nearly independent of flight time. A "180" geometry gives less dispersion, but energy shifts and changes in detection efficiency are large. X-rays produce small pulses with poor timing characteristics. As noted above, acceptance times for data acquisition are important; the " $90^{\circ}$ "geometry is more convenient for collimation to restrict acceptance times. A typical system for data acquisition is given in Fig. 2. In general these systems lacked versatility; only one event trigger could be active at a time. Energy spectra at moderate resolution [Si(Li) detector with Doppler spreading] but without mass selection are given in Fig. 3. If mass information is not needed, it is much better to stop both fragments in a low $Z$ absorber and obtain spectra limited only by detector resolution. However, with mass information such as shown in Fig. 4, correlations can be sought and spectrum analysis is more reliable (the continuum under the $\mathrm{X}$-ray peaks is well defined). Correcting $\mathrm{X}$-ray yield per fission for differences in fragment yields gives one of the most useful correlations, X-rays per fragment (see Fig. 5).

Which transitions lead to conversion? ATNEOSEN, THOMAS, GIBSON and PERLMAN ${ }^{8}$ measured spectra of conversion electrons $(30-500 \mathrm{keV})$ as a function of mass and repeated measurements of the times of emission of $X$-rays. They concluded the converted transitions were M1 or mixed M1-E2. SHAPIRO, WEHRING and WYMAN 21 measured the spectra of conversion electrons in coincidence with $X$-rays (with and without coincidence with fission). Their thin plastic scintillators used to detect fission prevented detection of low energy electrons or obtaining good energy 
H.C. GRIFFIN: THE EMISSION OF X-RAYS BY FISSION FRAGMENTS

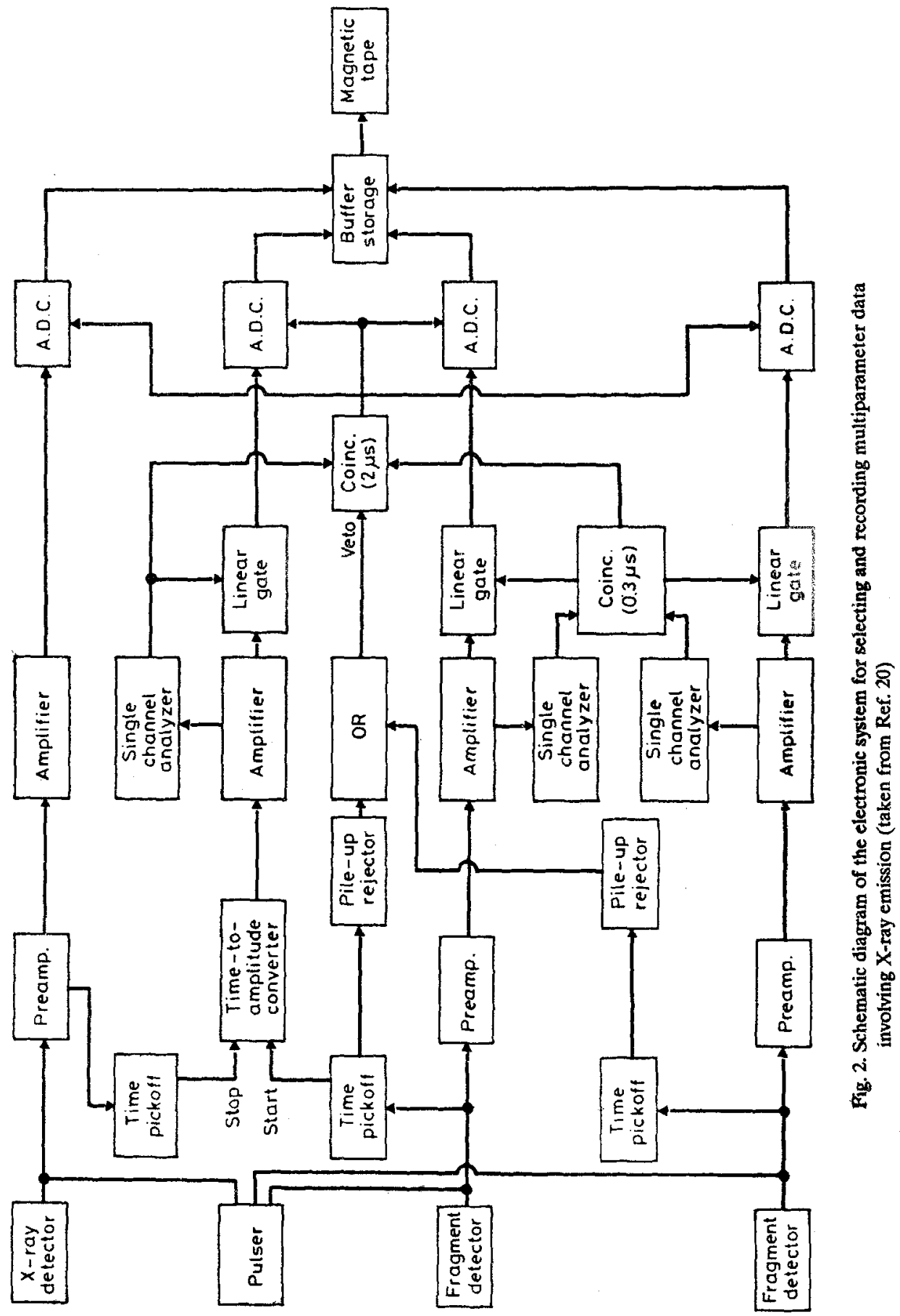




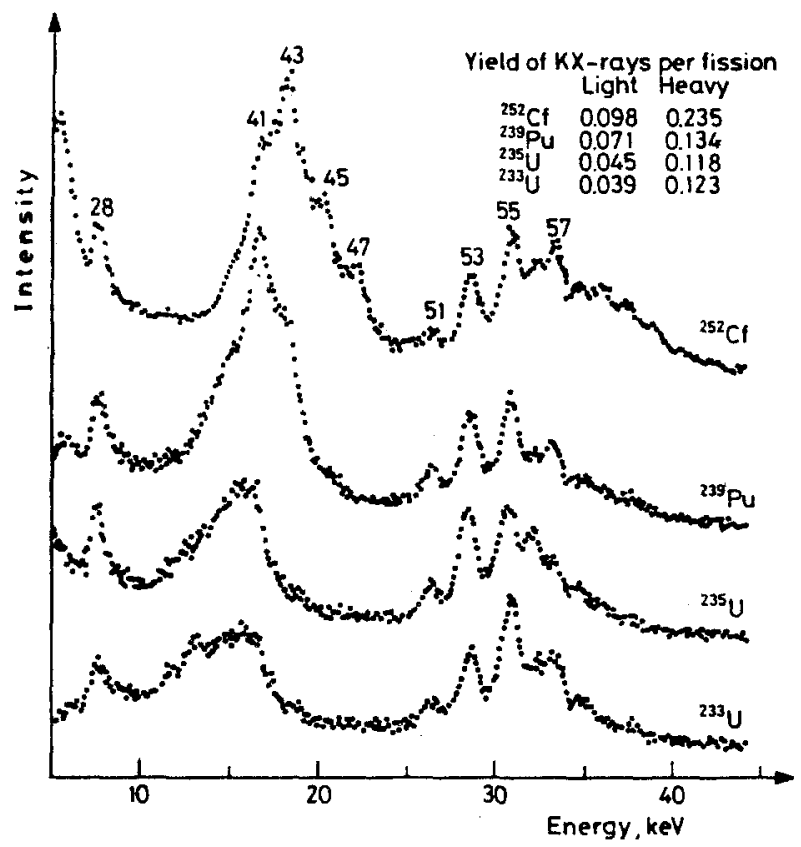

Fig. 3. Energy spectra of $\mathrm{KX}$-rays emitted within $1 \mathrm{~ns}$ after fission. Prominent peaks are identified by atomic numbers of the emitters. The $\mathrm{Ni}(\mathrm{Z}=28)$ peak arises in the supporting foils (taken from Ref. 16)

resolution. The earlier work by WATSON et al. ${ }^{12}$ remains the most thorough study of conversion electrons. They measured masses and X-ray energies, and element, and checks based on internal consistency showed that average properties were reliable. However, since no attention was given to individual nuclear structures or $\gamma$-ray patterns, details related to individual fission fragments could not be obtained.

Studies of the multiplicity of X-rays revealed features which were not accessible to other techniques. KAPOOR et al. ${ }^{13}$ used ${ }^{252} \mathrm{Cf}$, two Si detectors for X-rays, and a $2 \pi$ ionization chamber for fission detection. They recorded singles (X-rays in coincidence with fission) and doubles (a pair of $\mathrm{X}$-rays from fission). They found a strong probability for emission of two or more $\mathrm{X}$-rays, particularly for some heavy fragments. This supports the observation of WATSON et al. ${ }^{12}$ that X-ray emission is dominated by a few nuclides, including several odd-odd nuclides in the heavy group. ST. LAURENT, PHILLIPS, RICHARD and MOORE ${ }^{14}$ obtained similar results and noted that cascades within a fragment are more likely than coincidences between fragments. 

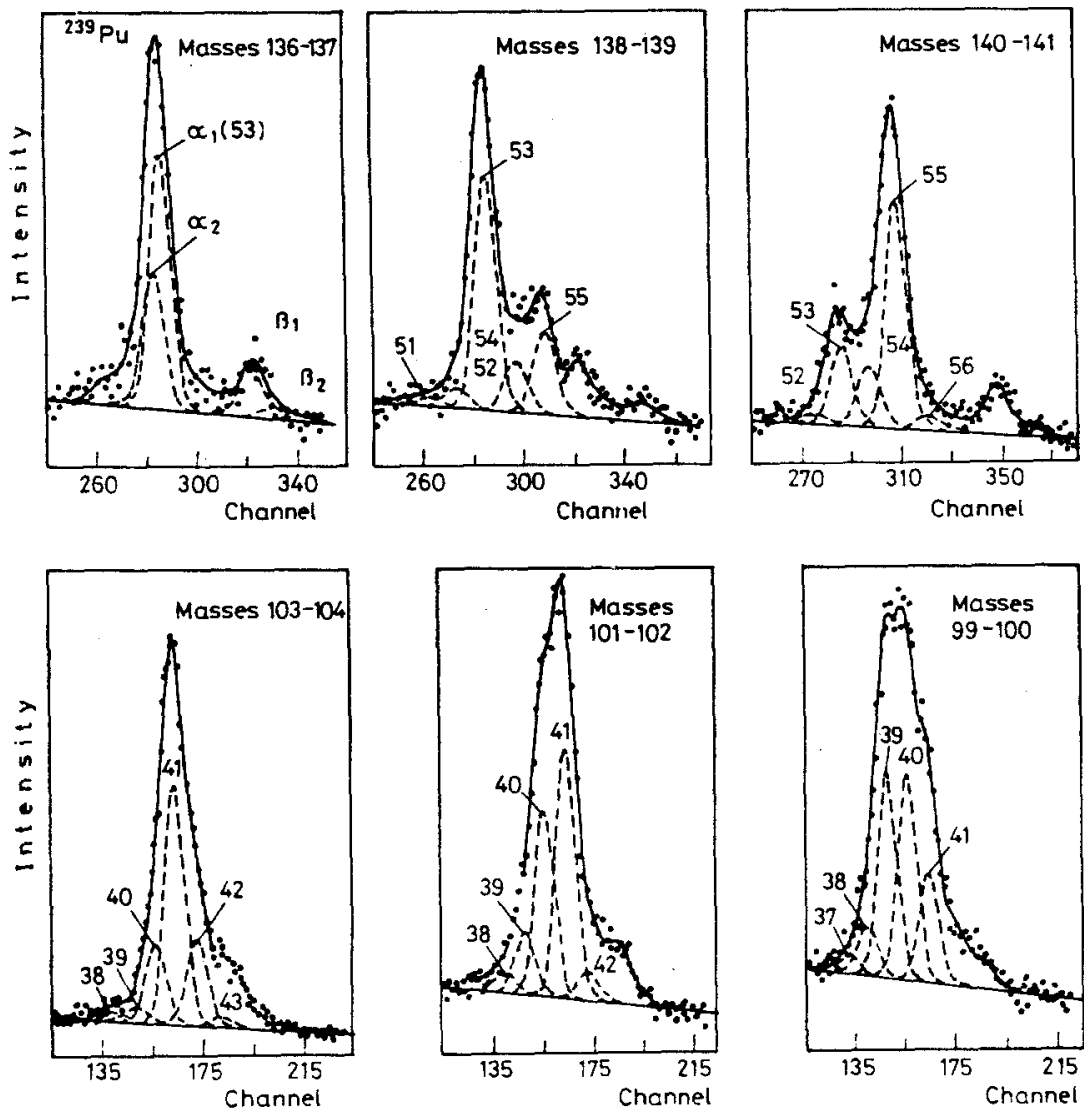

Fig. 4. Mass-sorted $\mathrm{K} \mathrm{X}$-ray spectra for adjacent complementary fragment mass intervals in thermal neutron fission of $2{ }^{29} \mathrm{Pu}$ (taken from Ref. 20)

This result is understandable since neutron emission spreads the masses of the complementary fragment to include nuclides with lower emission probabilities. Their data include transitions following $\beta$-decay, and interpretation in terms of fragment deexcitation is questionable. Still, the relatively high probability of $\mathrm{X}-\mathrm{X}$ coincidences compared with X-ray yield per fragment implies that X-ray emission is concentrated in a few fragments and most fragments emit very few $\mathrm{X}$-rays. 


\section{H.C. GRIFFIN: THE EMISSION OF X-RAYS BY FISSION FRAGMENTS}

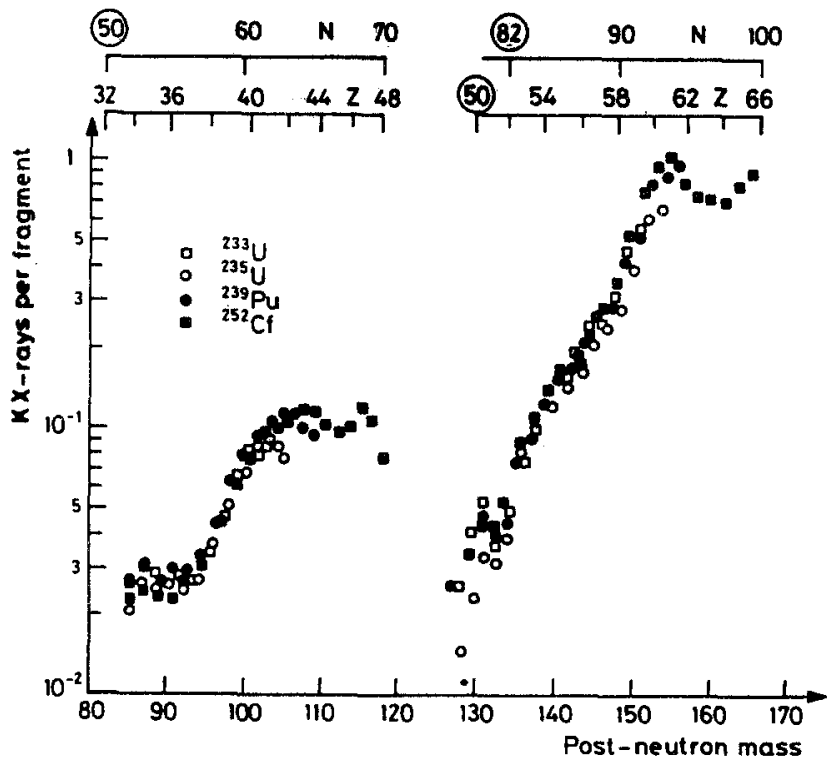

Fig. 5. K X-rays per fragment emitted within $1 \mathrm{~ns}$ after fission as a function of post-neutron mass. Approximate average proton and neutron numbers for ${ }^{252} \mathrm{Cf}$ fission are given (taken from Ref. 16)

NIFENECKER, SIGNARBIEUX, BABINET and PoITOU 25 have reviewed the gross features of neutron and $\gamma$-ray emission with an emphasis on correlations among $A$, $\nu, E_{\gamma}$ and $Z$. They report measurements in which high resolution $X$-ray spectra of stopped fragments are used to identify $Z$ in conjunction with neutron and $\gamma$-ray measurements. Again, the interpretation can be questioned in light of biases in the $X$-ray method.

As noted above, following 1972 there were few reports which explicitly treated Xrays emitted by fission fragments. One reason was noted by DONICKHIN, SMIRNOV and EISMONT ${ }^{26}$ - the easy measurements had been made. They measured the spectrum of $\mathrm{K} \mathrm{X}$-rays from fragments in fission of ${ }^{235} \mathrm{U}$ by $3.5-\mathrm{MeV}$ neutrons (from $\mathrm{d}+\mathrm{d}$ reactions at $0.5 \mathrm{MeV}$ ). In an hour their $\mathrm{Si}(\mathrm{Li})$ detector yielded only 300 coincidences with fragments from a singles rate (all pulse heights) of $3 \cdot 10^{4} \mathrm{~s}^{-1}$. Furthermore, radiation damage seriously degraded energy resolution. For all their effort, they found no differences from thermal neutron fission except effects from known shifts in fragment yields. Perhaps this reinforced the conclusion that little remained to be obtained from gross measurements of $\mathrm{X}$-rays in fission. The cumulative evidence was that $\mathrm{X}$-ray emission is dominated by a few transitions in odd 
( $Z$ and $N$ ) and odd-A nuclides. If one transition dominated (contrary to X-X coincidence measurements) $\mathrm{X}$-rays could be used to characterize that transition. If many transitions contributed (contrary to the large variations in yields for different nuclides), average properties could be revealed. Therefore X-ray spectroscopy remained useful primarily when individual transitions were relevant.

\section{Uses of X-rays}

A small fraction of the fissions of heavy nuclides at low excitation energies results in the emission of light charged particles (LCP) in addition to the two fragments. LCP are most often $\alpha$-particles. Radiochemical measurements cannot be used to investigate these process since most of the fissions are binary and chemistry measures cumulative properties. Event-by-event measurements can be used to first detect the ternary event and then determine characteristics. The emission of LCP is relatively easy to detect - they are more penetrating than fission fragments. For example, the angular distribution of LCP versus the fragment axis can be measured. Even with uncertainties in probabilities of emission, an X-ray detector can give atomic number information which would not otherwise be available (except by detecting $\gamma$-rays characteristics of individual nuclides as shown below). KAPOOR, RAMAMURTHY and RAMA RAO ${ }^{9}$ used three detectors (LCP, fission fragment, and X-ray) to investigate the origin of the LCP, which they assumed to be $\alpha$-particles. They concluded that LCP originate from the fissioning nucleus as a whole and are emitted just before scission. WATSON27 made similar measurements and concluded the technique was ambiguous. NIFENECKER ${ }^{28}$ attempted to correct for variations in X-ray emission probabilities by measuring neutron multiplicity simultaneously with X-ray energies (a questionable process in light of the dominance of $\mathrm{X}$-ray emission from a few nuclides); the data still were not adequate to decide among several models of LCP formation.

$\mathrm{X}$-rays can aid in assigning $\boldsymbol{\gamma}$-rays emitted by fission fragments to individual nuclides by giving element identification. RUEGSEGGER, EDDY and ROY11,29 used a single channel analyzer to select $\mathrm{K}_{\alpha} \mathrm{X}$-rays of individual elements $(\mathrm{Cs}, \mathrm{Ba}, \mathrm{La}, \mathrm{Ce}$, $\mathrm{Pr}$ ) and recorded coincident $\boldsymbol{\gamma}$-rays. The technique was greatly improved by HOPKINS, PHILLIPS, WHITE, MOORE and RICHARD, 30,31 but their data also included transitions following $\beta$-decay as well as fragment deexcitations. CHEIFETZ, WILHELMY, JARED and THOMPSON 15 have made the most effective use of the method by combining it with fission detection. mass measurements, and $\gamma-\gamma$ coincidence. They measured the intensities of $\gamma$ transitions in even-even fragments 


\section{H.C. GRIFFIN: THE EMISSION OF X-RAYS BY FISSION FRAGMENTS}

(confirmed by X-ray coincidences and mass measurements), showed that the $2^{+}$to $0^{+}$transitions are a good measure of fission yield, and infered charge distribution and dispersion for ${ }^{252} \mathrm{Cf}$ free from the systematic uncertainties of the X-ray method. They confirmed that even-even nuclides are not the main X-ray emitters.

PHILLIPS et al..$^{5}$ showed an appropriate use of quantitative $\mathrm{X}$-ray measurements in a detailed study of the structure of ${ }^{144} \mathrm{Ba}$. This nuclide was expected to have relatively stable reflection-asymetric shapes, which could lead to interlaced positive- and negative-parity levels. About $3.5 \%$ of the fissions of ${ }^{252} \mathrm{Cf}$ produce ${ }^{144} \mathrm{Ba}$. In spite of relatively low average spin of fission fragments (about $5 \hbar$ ), transitions in the main band were observed up to spin $I=15$. This sensitivity obtained from an array of seven bismuth-germanate-suppressed Ge detectors. A small, high-resolution $\mathrm{Ge}$ detector was used for detecting low energy transitions, including X-rays. Angular correlations (showing dipole character), conversion coefficients (from X-ray measurements for the lowest energy transition suspected as E1), and branching ratios (used to infer absolute transition probabilities) were all required to give confidence to the conclusion that the interlaced levels did have opposite parity.

\section{Conclusions}

X-rays emitted by fission fragments can be used to quantify internal conversion or as a qualitative (perhaps semiquantitative) measure of atomic number.

\section{References}

1. V.K. VotTovetski, B.A. LeVIn, E.V. Marchenko, Soviet Phys. JETP, 5 (1957) 184.

2. V.V. Skitarevski, D.E. Fomenko, E.P. Stepanov, Soviet Phys. JETP, 5 (1957) 220.

3. R.B. Lechmann, Proc. Int. Conf. Peaceful Uses of Atomic Energy 15, United Nations, Geneva, 1958, p. 331, and R.E. Carter, J.J. Wagner, M. E. Wyman, Bull. Am. Phys. Soc., 3 (1958) 228.

4. L.E. Glendenin, H.C. GRifFin, Phys. Lett., 15 (1965) 153.

5. W.R. Phillips, I. Ahmad, H.Emling, R. Holzmann, R.V.F. Janssens, T.-L. Khoo, M.W. Drigert, Phys. Rev. Lett., 57 (1986) 3257.

6. L.E. Glendenin, J.P. Unik, Phys. Rev., 140 (1965) B1301.

7. S.S.KAPOOR, H.R. Bowman, S.G. ThOMPSON, Phys. Rev., 140 (1965) B1310.

8. R.A. Atneosen, T.D. Thomas, W.M. Gibson, M.L. Perlman, Phys. Rev, 148 (1966) 1206.

9. S.S.Kapoor, V.S. Ramamurthy, P.N. Rama Rao, Phys. Rev., 166 (1968) 1190.

10. E.M. Bohn, B.W. Wehring, M.E. Wyman, Phys. Rev., 188 (1969) 1909.

11. D.R. Ruegsegger, R.R. Roy, Phys. Rev., C1 (1970) 631.

12. R.L. Watson, J.B. Wilhelmy, R.C. Jared, C. Rugge, H.R. Bowman, S.G. Thompson, J.O. Rasmussen, Nucl. Phys., A141 (1970) 449.

13. S.S. KAPOOR, S.K. KATARIA, S.R.S. MURTHY, D.M. NADKARNi, V.S. RAMAMURTHY, P.N. RAMA RAO, Phys. Rev., C4 (1971) 2165. 
14. R.St.-Laurent, G.W. Philuips, P. Richard, C.F. Moore, Phys. Rev., C4 (197i) 1948.

15. E. Cheifetz, J.B. Wilhelmy, R.C. JARed, S.G. Thompson, Phys. Rev., C4 (1971) 1913.

16. W. Reisdorf, J.P. UNik, H.C. Griffin, L.E. Glendenin, Nucl. Phys., A177 (1971) 337.

17. N. BoHR, Phys. Rev., 59 (1941) 270.

18. A.H. SNeLl, in Alpha-,Beta- and Gamma-ray Specroscopy, K. Siegrahn, (Ed.) North-Holland, Amsterdam, 1968.

19. A.B. Smith, P.R. Fields, A.M. Friedman, Phys. Rev., 104 (1956) 699.

20. L.E. Glendenin, H.C. GRIFFin, W. Reisdorf, J.P. UniK, in Physics and Chemistry of Fission, IAEA, Vienna, 1969.

21. N.L. Shapiro, B.W.Wehring, M.E. Wyman, Phys. Rev., C3 (1971) 2464.

22. W. John, F.W. GuY, J.J. Wesolowsky, Phys. Rev., C2 (1970) 1451.

23. R.G. Clark, L.E. Glendenin, W.L. Talbert, Jr., in Physics and Chemistry of Fission, 1973, Vol. II, IAEA, Vienna, 1974.

24. R.L. Watson, R.C. JAREd, S.G. Thompson, Phys. Rev., C1 (1970) 1866.

25. H. Nifenecker, C. Signarbieux, R Babinet, J. Portou, in Physics and Chemistry of Fission, 1973, Vol. II, IAEA, Vienna, 1974.

26. A.G. Donichkin, A.N. Smirnov, V.P. Eismont, Sov. J. Nucl. Phys., 25 (1977) 19.

27. RL. Watson, Phys. Rev., 179 (1969) 1109.

28. H. NifENECKER. in Physics and Chemistry of Fission, 1973, Vol. II, IAEA, Vienna, 1974.

29. N.W. EDDY, R.R. RoY, Phys. Rev., C3 (1971) 877.

30. F.F. Hopkins, G.W. Philups, J.R. White, C.F. Moore, P. Richard, Phys. Rev., C4 (1971) 1927.

31. F.F. Hopkins, J.R. White, G.W. Philups, C.F. Moore, P. Richard, Phys. Rev., C5 (1972) 1015. 